\title{
No Evidence of Association of the Arg72Pro p53 Gene Polymorphism with Cancer Risk in the Saudi Population: a Meta-Analysis
}

\author{
Mohammad Irshad ${ }^{1}$, Raju Kumar Mandal ${ }^{1}$, Abdulmajeed Al-Drees ${ }^{1}$, Mahmoud \\ Salah Khalil ${ }^{1,2}$, Hamza Mohammad Abdulghani ${ }^{1 *}$
}

\begin{abstract}
Background: Earlier studies on the association between p53 codon $72 \mathrm{Arg}>$ Pro polymorphism and cancer risk were inconclusive and conflicting for the Saudi population. Therefore, we performed a meta-analysis to investigate the relationship between the codon $72 \mathrm{Arg}>$ Pro polymorphism and overall cancer risk in Saudi Arabia. Materials and Methods: We searched all eligible published studies and data were pooled together to perform the meta-analysis. Pooled odds ratios (ORs) and 95\% confidence intervals (95\% CIs) were calculated for homozygous, heterozygous, dominant and recessive genetic models. Results: A total of five eligible published studies covering 502 cancer cases and 784 healthy controls were included in the meta-analysis. No publication bias was detected in this study. The results suggested that the variant (Pro vs Arg: $\mathrm{p}=0.960 ;$ OR=1.004, 95\% $\mathrm{CI}=0.852-1.183$ ), homozygous (Pro.Pro vs Arg.Arg: $\mathrm{p=0.970;} \mathrm{OR=1.006,95 \%} \mathrm{CI}=\mathbf{0 . 7 2 9 - 1 . 3 9 0}$ ), heterozygous (Arg.Pro vs Arg.Arg: $\mathrm{p}=\mathbf{0 . 4 7 3}$; $\mathrm{OR=0.783,95 \%} \mathrm{CI}=\mathbf{0 . 4 0 2 - 1 . 5 2 7}$ ) carriers were not associated with overall cancer risk. Similarly, dominant (Pro.Pro+Pro.Arg $v$ s Arg.Arg: $\mathrm{p}=0.632 ; \mathrm{OR}=0.886,95 \% \mathrm{CI}=\mathbf{0 . 5 4 0 - 1 . 4 5 4 )}$ ) and recessive (Pro.Pro vs Pro.Arg+Arg.Arg: $\mathrm{p}=\mathbf{0 . 2 6 9}$; $\mathrm{OR=1.163,95 \%} \mathrm{CI}=\mathbf{0 . 8 9 0 - 1 . 5 2 1}$ ) models also did not indicate increased risk of cancer. Conclusions: The current meta-analysis suggests that the codon $72 \mathrm{Arg}>$ Pro polymorphism of the 533 gene might not contribute to cancer susceptibility in Saudi population. Future well designed large case control studies are needed to validate our findings.
\end{abstract}

Keywords: Meta-analysis - p53 polymorphism - cancer - Saudi Arabia

Asian Pac J Cancer Prev, 16 (14), 5663-5667

\section{Introduction}

Cancer is a most dreadful disease for humankind and leading cause of death worldwide (Jemal et al., 2011). Despite its low incidence rate as compared to western countries, it still continues to be a major problem in Saudi Arabia (Saudi Cancer Registry, 2014). Cancer is a multifactorial disease and its incidence rate varies among worldwide due to variation of geographical region, habitats and genomic frequency (Pharoah et al., 2004). Epidemiological studies suggest that the majority of cancers is polygenic and several genes with modest effect are involved in development of carcinogenesis (Vineis, 2004; Verit and Yucel, 2013). Thus, identification of genetic risk markers related to cancer risk is important, as it may allow for the creation of early diagnostic factors for individual and population risks, and helps in the understanding of pathophysiological mechanisms of carcinogenesis.
TP53 protein, encoded by p53 gene (TP53 at 17p13) is a tumor suppressor gene which is involved in various cellular processes, such as cell cycle arrest, senescence, apoptosis, inhibition of tumorigenesis and protects the genomic integrity (Suzuki and Matsubara, 2011; AlFatlawi et al., 2014). p53 gene is mutated in 50-70\% in human cancers (Suzuki and Matsubara, 2011; Shin and Kim, 2014), and alter expression of p53 in serum is associated with various malignant tumors (Rivlin et al., 2011; Dunna et al., 2012), which highlights the significance role of p53 in malignancy.

During past decades, numbers of genetic polymorphism in p53 locus have been widely investigated fo their effect in different cancer risks (Liu et al., 2014; Rao et al., 2014; Pouladi et al., 2014), among them the most common polymorphism is at codon 72 (Arg72Pro) $(\mathrm{G}>\mathrm{C})$ (rs1042522) This codon which is found at exon 4 of the p53 gene and is frequently studied worldwide (Vijayaraman et al., 2012; Xiang et al., 2012; Kafshdooz

${ }^{1}$ Department of Medical Education, College of Medicine, King Saud University, Riyadh, Saudi Arabia ${ }^{2}$ Department of Medical Education, College of Medicine King, Saud University, and Department of Histology, Faculty of Medicine, Suez Canal University, Ismailia,Egypt*For correspondence: hamzaabg@gmail.com; hamzaab@ksu.edu.sa 
et al., 2014). At codon 72, arginine to proline substitution altered the p53 gene translation product, resulted in a reduced capacity of DNA repair, cell cycle regulation, apoptosis and thereby increased the susceptibility of cancer risk (Grochola et al., 2010).

The significance of p53 gene in carcinogenesis, has considered codon 72 Arg>Pro polymorphism, could be a potential predictive marker for prevention and early intervention of cancers (Hrstka et al., 2009). Also, genetic variant of p53 gene appears as good resources to study inter individual differences in cancer risk and therapeutic response (Lin et al., 2008).

Many studies have investigated the role of p53 codon $72 \mathrm{Arg}>$ Pro polymorphism in many types of cancers in Saudi population, but the results is remaining inconclusive or inconsistent (Siraj et al., 2008; Alshatwi et al., 2012; AlHadyan et al., 2012; Al-Qasem et al., 2012; Alsbeih et al., 2013). An individual study might have small sample size and not be powered sufficiently to detect a small effect of this polymorphism on cancer susceptibility. Meta-analysis is a powerful tool to combine the data from individual studies and provide robust conclusion (Mandal et al., 2013). Therefore, we performed a meta-analysis from all eligible case-control studies to clarify the relationship between $\mathrm{p} 53$ codon $72 \mathrm{Arg}>$ Pro polymorphism and cancer risk in Saudi population.

\section{Materials and Methods}

\section{Identification and eligibility of relevant studies}

We have followed "PRISMA" 2009 checklist criteria for meta-analysis. We carried out a PubMed (Medline), EMbase, Cochrane, Google (scholar) web database search covering all research articles published (including personal communication) using following key words, "p53 gene polymorphism”, "p53 gene mutation”, "p53 gene variant" and "cancer", "carcinoma", "risk", "tumor susceptibility" in Saudi population (last updated on December 2014). All the searched studies were retrieved and the reference lists were checked for any other relevant studies.

\section{Inclusion and exclusion criteria}

To minimize the bias and heterogeneity to interpretation of our results, studies included in the current meta-analysis had to meet all the following criteria: (i) evaluation of the p53 codon 72 Arg>Pro, and cancer risk, (ii) use of a case-control design, (iii) recruitment of pathological confirmed cancer patients and cancer-free controls, (iv) and the available genotype frequency. Additionally, when the case-control study was included by more than one article using the same case series, we selected the study that included the largest number of individuals. The major criteria for exclusion of studies were $(i)$ overlapping data and (ii) only case reported studies, (iii) genotype frequency missing, (iv) family based studies, and (v) review articles.

\section{Data extraction and quality assessment}

For each publication, the methodological quality assessment and data extraction were independently abstracted in duplicate using a standard protocol and according to the inclusion criteria by two independent authors (MI and RKM). Disagreement between the authors was resolved after discussion among the authors. The following information was sought from each article: the year of publication, the number of cases and controls, type of study, genotype frequencies, and the Hardy-Weinberg (HWE).

\section{Statistical analysis}

We calculated the odds ratio (OR) and corresponding 95\% confidence interval (CI), to evaluate the association between the p53 codon 72 Arg>Pro polymorphism and cancer risk. Heterogeneity assumption was checked by the chi-square-based Q-test ( Wu and Li, 1999). A p-value $(<0.05)$ for the Q-test indicates a lack of heterogeneity among the studies, and then the pooled OR was calculated by the fixed effects model (Mantel and Haenszel, 1959); otherwise, the random-effects model was used (DerSimonian and Laird, 1986). In addition, $\mathrm{I}^{2}$ statistics was used to quantify inter study variability. It ranges between $0 \%$ and $100 \%$, where a value of $0 \%$ indicates no observed heterogeneity, and larger values indicate an increasing degree of heterogeneity (Higgins et al., 2003). The HWE was examined in the control subjects using a goodness-of-fit chi-square test for each study. Publication bias was assessed by visual inspection of funnel plots in which the standard error of $\log (\mathrm{OR})$ of each study was plotted against its log (OR). An asymmetric plot suggests a possible publication bias. Funnel plot asymmetry was also assessed by the Egger's linear regression test. The significance of the intercept was determined by the t-test $(p<0.05)$ which was considered representative of statistically significant publication bias (Egger et al., 1997). All statistical analysis for meta-analysis was performed by comprehensive meta-analysis (CMA) V2 software (Biostat). CMA V2 has several advantages over other software available for computing meta-analyses (http://metaanalysis. com/pages/comparisons.html).

\section{Results}

\section{Characteristics of published studies}

Nineteen articles were recovered by literature search. All retrieved articles were reviewed by reading the title, abstract and the full texts for the potentially relevant publications and further checked for their suitability for this meta-analysis. We excluded 14 articles because their study designs were not matched with our inclusion criteria (Figure 1). Studies using the p53 codon 72 Arg $>$ Pro polymorphism to predict survival risk in cancer or considering indicators for response to therapy were excluded. Studies to investigate the levels of p53 mRNA or p53 codon $72 \mathrm{Arg}>$ Pro protein expression were also excluded. We included only case-control studies. After careful screening, finally five eligible published studies were included in this study (Table 1). The distribution of genotypes, MAF and HWE is tabulated in Table 2.

\section{Evaluation of potential publication bias}

The Begg's funnel plot and Egger's test were performed to access the publication bias of literatures included for meta-analysis. The shape of funnel plots and 
Lack of Association of the Arg72Pro of 533 Gene Polymorphism with Cancer Risk in the Saudi Population: A Meta-Analysis Table 1. Main Characteristics of All Five Studies Included In The Meta-analysis

\begin{tabular}{|c|c|c|c|c|c|}
\hline Authors and year & Types of cancer & Study design & Genotyping method & Control & Cases \\
\hline Alsbeih et al., 2013 & Cervical & $\mathrm{HB}$ & Sequencing & 100 & 100 \\
\hline Alshatwi et al., 2012 & Breast & $\mathrm{HB}$ & Taq Man & 100 & 100 \\
\hline Al-Hadyan et al., 2012 & Head and Neck & HB & Sequencing & 251 & 156 \\
\hline Al-Qasem et al., 2012 & Breast & $\mathrm{HB}$ & Sequencing & 108 & 100 \\
\hline Siraj et al., 2008 & Papillary thyroid & $\mathrm{HB}$ & PCR-RFLP & 225 & 46 \\
\hline
\end{tabular}

Table 2. Genotypic Distribution of p53 (72) Gene Polymorphism Included in Meta-analysis

\begin{tabular}{|c|c|c|c|c|c|c|c|c|c|}
\hline \multirow[t]{3}{*}{ Authors and year } & \multicolumn{4}{|c|}{ Controls } & \multicolumn{4}{|c|}{ Cancer cases } & \multirow{3}{*}{$\frac{\text { HWE }}{\text { p value }}$} \\
\hline & \multicolumn{3}{|c|}{ Genotype } & \multirow{2}{*}{$\frac{\text { Minor allele }}{\text { MAF }}$} & \multicolumn{3}{|c|}{ Genotype } & \multirow{2}{*}{$\begin{array}{c}\text { Minor allele } \\
\text { MAF }\end{array}$} & \\
\hline & $\begin{array}{c}\text { GG } \\
\text { Arg.Arg }\end{array}$ & $\begin{array}{c}\text { GC } \\
\text { Arg.Pro }\end{array}$ & $\begin{array}{c}\mathrm{CC} \\
\text { Pro.Pro }\end{array}$ & & $\begin{array}{c}\text { GG } \\
\text { Arg.Arg }\end{array}$ & $\begin{array}{c}\text { GC } \\
\text { Arg.Pro }\end{array}$ & $\begin{array}{c}\mathrm{CC} \\
\text { Pro.Pro }\end{array}$ & & \\
\hline Alsbeih et al., 2013 & 22 & 52 & 26 & 0.52 & 20 & 58 & 22 & 0.51 & 0.67 \\
\hline Alshatwi et al., 2012 & 32 & 51 & 17 & 0.42 & 22 & 52 & 26 & 0.52 & 0.66 \\
\hline Al-Hadyan et al., 2012 & 71 & 110 & 70 & 0.49 & 48 & 70 & 38 & 0.46 & 0.05 \\
\hline Al-Qasem et al., 2012 & 19 & 65 & 24 & 0.52 & 39 & 25 & 36 & 0.48 & 0.03 \\
\hline Siraj et al., 2008 & 68 & 105 & 52 & 0.46 & 14 & 19 & 13 & 0.48 & 0.35 \\
\hline
\end{tabular}

*MAF, Minor allele frequency, HWE, Hardy Weinberg equilibrium

Table 3. Statistics to Test Publication Bias and Heterogeneity in Meta-analysis

\begin{tabular}{|c|c|c|c|c|c|c|c|}
\hline \multirow[t]{2}{*}{ Comparisons } & \multicolumn{3}{|c|}{ Egger's regression analysis } & \multicolumn{3}{|c|}{ Heterogeneity analysis } & \multirow{2}{*}{$\begin{array}{l}\text { Models for } \\
\text { meta-analysis }\end{array}$} \\
\hline & Intercept & $95 \% \mathrm{CI}$ & $\mathrm{p}$ & $\mathrm{Q}$ value & Pheterogeneity & $\mathrm{I}^{2}(\%)$ & \\
\hline Pro $v s$ Arg & 3.12 & -19.86 & 0.39 & 5.13 & 0.27 & 22.09 & Fixed \\
\hline Pro/Pro vs Arg/Arg & 3.03 & -17.31 & 0.34 & 5.21 & 0.26 & 23.25 & Fixed \\
\hline Arg/Pro vs Arg/Arg & -2.53 & -40 & 0.71 & 20.93 & $<0.0001$ & 80.89 & Random \\
\hline Pro/Pro+Arg/Pro vs Arg/Arg & 0.25 & -32.7 & 0.96 & 13.41 & 0.009 & 70.18 & Random \\
\hline Pro/Pro vs Arg/Arg+Arg/Pro & 4.05 & -22.08 & 0.32 & 7.48 & 0.11 & 46.56 & Fixed \\
\hline
\end{tabular}
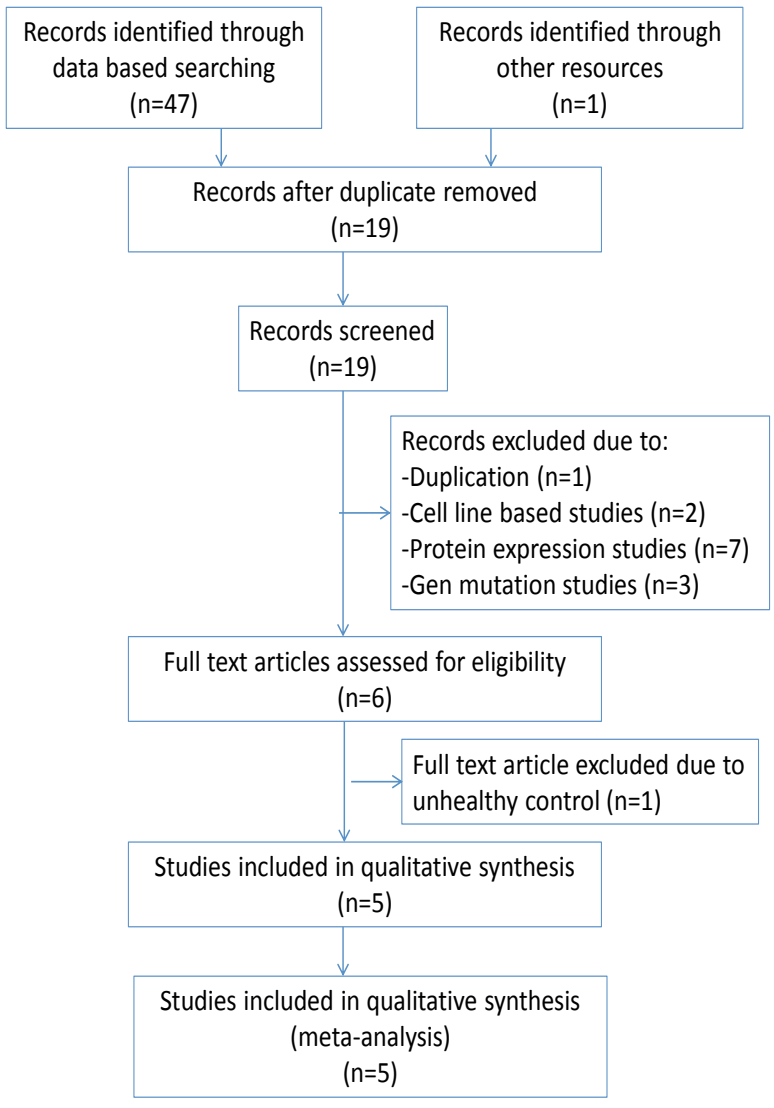

Figure 1. Flow Diagram of the Study Selection Process

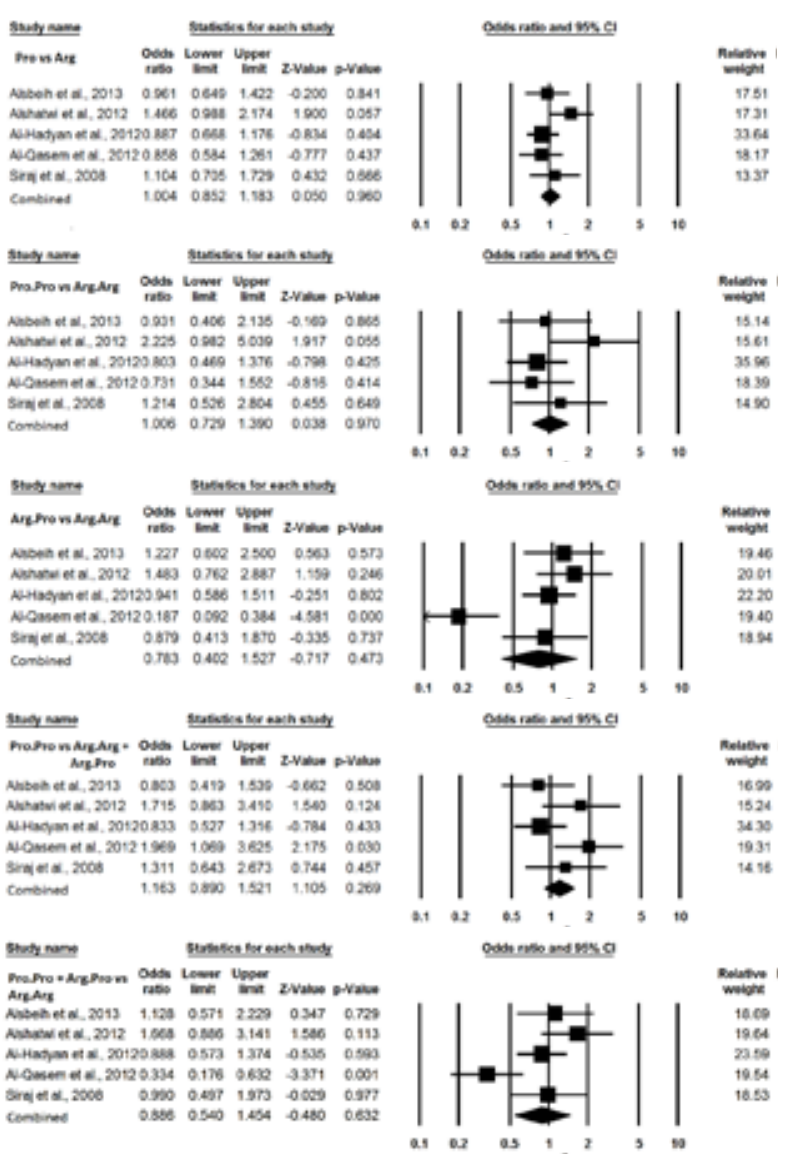

Figure 2. Genetic Modeling and Risk for Cancer 
Egger's test did not show any evidence of publication bias in our meta-analysis (Table 3).

Test of heterogeneity

Heterogeneity was observed in two genetic models, heterozygous (GC vs GG) and dominant (CC+GC vs GG) which were included for the analysis. Hence, the random effects model was used for calculation of OR and 95\%CI.

Association of 53 codon 72 Arg $>$ Pro gene polymorphism and overall cancer susceptibility

We pooled all the five studies together, which comprise of 784 controls and 502 cancer cases to assess the overall association between the p53 codon 72 Arg $>$ Pro polymorphism and cancer risk. The results indicated that codon 72 Arg $>$ Pro polymorphism might not be associated with on increased or decreased risk of developing cancer among Saudi population in all analyzed genetic models, such as allelic (Pro vs Arg: $\mathrm{p}=0.960 ; \mathrm{OR}=1.004$, 95\% $\mathrm{CI}=0.852-1.183$ ), heterozygous (Arg.Pro vs Arg. Arg: $\mathrm{p}=0.473$; $\mathrm{OR}=0.783,95 \% \mathrm{CI}=0.402-1.527)$ and homozygous (Pro.Pro vs Arg.Arg: $\mathrm{p}=0.970$; $\mathrm{OR}=1.006$, 95\% CI=0.729-1.390) comparisons. Similarly, dominant (Pro.Pro+Arg.Pro vs Arg.Arg: $\mathrm{p}=0.632 ; \mathrm{OR}=0.886,95 \%$ $\mathrm{CI}=0.540-1.454$ ) and recessive (Pro.Pro $v s$ Arg.Pro+Arg. Arg: $\mathrm{p}=0.269 ; \mathrm{OR}=1.163,95 \% \mathrm{CI}=0.890-1.521)$ genetic models also were not associated with an increased risk of developing cancer (Figure 2).

\section{Discussion}

During last decades, cancers researcher has led interest to the association between single nucleotide polymorphism (SNPs) and cancer susceptibility (Alsbeih et al., 2010; Nassiri et al., 2013). P53 gene is a multifunctional tetrameric transcription factor involved in many important biological processes (Levine and Oren, 2009). The codon 72 polymorphism, the most common SNP in the TP53 gene, located in the non-conserved proline-rich region of exon 4 (Aizat et al., 2011). This polymorphism encodes either arginine (CGC) or proline (CCC) and is functionally important in growth suppression and apoptosis (Katkoori et al., 2009). The frequency of this polymorphism also varies among different races and ethnic groups (Murphy, 2006). Inter-individual variation in apoptotic capacity is largely attributed to an individual's genetic constitution, and genetic variation of apoptosis related genes may affect the expression of proteins and associated with the risk and prognosis of many cancers (Pathak et al., 2014).

Given the important roles of p53 in carcinogenesis, it is reasonable to speculate that host genomic polymorphism of p53 gene may affect the tumor occurrence. Recently, Genetic variants of the p53 codon 72 Arg>Pro polymorphism and its role in the etiology of several cancers have been studied extensively in Saudi population, but the results are inconclusive.

Among the five eligible studies, two studies reported statistical significance risk association of Pro allele of p53 codon 72 in breast and head and neck cancer respectively (Alshatwi et al., 2012; Al-Hadyan et al., 2012). The present meta-analysis results indicated that p53 codon $72 \mathrm{Arg}>$ Pro polymorphism did not have statistically significant relationship between overall cancer susceptibility in Saudi population. Individual carrying the Pro allele did not exhibit any risk of cancer in all the eligible genetic models, when compared with individual carrying the Arg allele. Thus, it is possible that codon 72 Arg $>$ Pro analyzed variant do not influence individual cancer risk. Similar to our result, no association was reported in meta-analysis of p53 codon $72 \mathrm{Arg}>$ Pro polymorphism and risk of cervical cancer (Zhou et al., 2012), breast cancer (Hou et al., 2013), head and neck squamous cell carcinoma (Xia et al., 2012; Ren et al., 2014). Although, recently significant association was reported Arg $>$ Pro polymorphism in thyroid carcinoma under the recessive model in Asian population (Wang et al., 2014). Cancer is a multistep process and a single genetic variant is usually insufficient to predict risk of this deadly disease that has a complex disease phenotype. Gene polymorphisms are complicated and fluctuating, which mainly attributed to different ethnicities.

There are some limitations to our meta-analysis that should be considered when interpreting the results. First, present assessment was based on unadjusted estimates because of data limitations. Second, the number of studies included in the analysis was relatively small. Third, gene-gene and gene-environment interaction was not performed.

Despite these limitations, however, our meta-analysis has important advantages. First, the substantial number of cases and controls are included in this meta-analysis significantly increased the statistical power of this analysis as compare to individual study. Second, we did not have publication bias in the included studies, which possibly, suggest the reliability of our results.

In conclusion, our meta-analysis evaluated the relationship between the p53 codon 72 Arg>Pro polymorphism and cancer risk and revealed that the p53 codon 72 Arg $>$ Pro polymorphism might not statistically modulate the overall cancer risk in Saudi population. Further larger studies are warranted to clarify the role of this polymorphism in the pathophysiology of cancer.

\section{Acknowledgements}

This work was funded by the College of Medicine Research Center, Deanship of Scientific Research, King Saud University, Riyadh, Saudi Arabia.

\section{References}

Aizat AA, Shahpudin SN, Mustapha MA, et al (2011). Association of Arg72Pro of P53 polymorphism with colorectal cancer susceptibility risk in Malaysian population. Asian Pac J Cancer Prev, 12, 2909-13.

Al-Fatlawi AA, Al-Fatlawi AA, Irshad M, et al (2014). Rice bran phytic acid induced apoptosis through regulation of Bcl-2/Bax and p53 genesin HepG2 human hepatocellular carcinoma cells. Asian Pac J Cancer Prev, 15, 3731-6.

Al-Hadyan KS, Al-Harbi NM, Al-Qahtani SS, et al (2012). Involvement of single-nucleotide polymorphisms in predisposition to head and neck cancer in Saudi Arabia. Genet Test Mol Biomarkers, 16, 95-101.

Al-Qasem A, Toulimat M, Tulbah A, et al (2012). The p53 codon 
72 polymorphism is associated with risk and early onset of breast cancer among Saudi women. Oncol Lett, 3, 875-8.

Alsbeih G, Al-Harbi N, Al-Hadyan K, et al (2010). Association between normal tissue complications after radiotherapy and polymorphic variations in TGFB 1 and $\mathrm{XRCC} 1$ genes. Radiat Res, 173, 505-11.

Alsbeih G, Al-Harbi N, El-Sebaie M, et al (2013). HPV prevalence and genetic predisposition to cervical cancer in Saudi Arabia. Infect Agent Cancer, 8, 1-15.

Alshatwi AA, Hasan TN, Shafi G (2012). A single-nucleotide polymorphism in the TP53 and MDM-2 gene modifies breast cancer risk in an ethnic Arab population. Fundam Clin Pharmacol, 26, 438-43.

Saudi Cancer Registry (2014). http://www.scr.org.sa

DerSimonian R, Laird N (1986). Meta-analysis in clinical trials. Control Clin Trials, 7, 177-88.

Dunna NR, Vure S, Sailaja K, et al (2012). TP53 codon 72 polymorphism and risk of acute leukemia. Asian Pac J Cancer Prev, 13, 347-50.

Egger M, Davey-Smith G, Schneider M, et al (1997). Bias in meta-analysis detected by a simple, graphical test. $B M J$, 315, 629-34.

Grochola LF, Zeron-Medina J, Meriaux S, et al (2010). Singlenucleotide polymorphisms in the p53 signaling pathway. Cold Spring Harb Perspect Biol, 2, a001032.

Higgins JP, Thompson SG, Deeks JJ (2003). Measuring inconsistency in meta-analyses. $B M J, 327,557-60$.

Hou J, Jiang Y, Tang W, et al (2013) p53 codon 72 polymorphism and breast cancer risk: A meta-analysis. Exp Ther Med, 5, 1397-402.

Hrstka R, Coates PJ, Vojtesek B (2009). Polymorphisms in p53 and the 553 pathway: roles in cancer susceptibility and response to treatment. J Cell Mol Med, 13, 440-53.

Jemal A, Bray F, Center MM (2011). Global cancer statistics. CA Cancer J Clin, 61, 69-90.

Kafshdooz T, Tabrizi AD, Mohaddes Ardabili SM, et al (2014). Polymorphism of p53 gene codon 72 in endometrial cancer: correlation with tumor grade and histological type. Asian Pac J Cancer Prev, 15, 9603-6.

Katkoori VR, Jia X, Shanmugam C, et al (2009). Prognostic significance of $\mathrm{p} 53$ codon 72 polymorphism differs with race in colorectal adenocarcinoma. Clin Cancer Res, 15, 2406-16.

Levine AJ, Oren M (2009). The first 30 years of p53: growing ever more complex. Nat Rev Cancer, 9, 749-58.

Lin HY, Huang CH, Wu WJ, et al (2008). TP53 codon 72 gene polymorphism paradox in associated with various carcinoma incidences, invasiveness and chemotherapy responses. Int $J$ Biomed Sci, 4, 248-54.

Liu L, Zhang D, Jiao JH, et al (2014). Association between the TP53BP1 rs2602141 A/C polymorphism and cancer risk: a systematic review and meta-analysis. Asian Pac J Cancer Prev, 15, 2917-22.

Mandal RK, Yaday SS, Panda AK, et al (2013). Insertion/ deletion polymorphism of the ACE gene increased risk of Behcet disease: evidence from a meta-analysis. Ann Saudi Med, 33, 437-42.

Mantel N, Haenszel W (1959). Statistical aspects of the analysis of data from retrospective studies of disease. J Natl Cancer Inst, 22, 719-48.

Murphy ME (2006). Polymorphic variants in the p53 pathway. Cell Death Differ, 13, 916-20.

Nassiri M, Kooshyar MM, Roudbar Z, et al (2013). Genes and SNPs associated with non-hereditary and hereditary colorectal cancer. Asian Pac J Cancer Prev, 14, 5609-14.

Pathak A, Wenzlaff AS, Hyland PL, et al (2014). Apoptosisrelated Single Nucleotide Polymorphisms and the risk of non-small cell lung cancer in women. J Cancer Ther Res, $\mathbf{3}$.
Pharoah PD, Dunning AM, Ponder BA, et al (2004). Association studies for finding cancer-susceptibility genetic variants. Nat Rev Cancer, 4, 850-60.

Pouladi N, Kouhsari SM, Feizi MH, et al (2014). Lack of association of intron $316 \mathrm{bp}$ polymorphism of TP53 with breast cancer among Iranian-Azeri patients. Asian Pac J Cancer Prev, 15, 2631-4.

Rao AK, Vinothkumar V, Revathidevi S, et al (2014). Absence of the TP53 Poly-A signal sequence variant rs78378222 in oral, cervical and breast cancers in south India. Asian Pac $J$ Cancer Prev, 15, 9555-6.

Rivlin N, Brosh R, Oren M, et al (2011). Mutations in the p53 tumor suppressor gene important milestones at the various steps of tumorigenesis. Genes Cancer, 2, 466-74.

Shin MK, Kim JW (2014). Clinicopathologic and diagnostic significance of $\mathrm{p} 53$ protein expression in papillary thyroid carcinoma. Asian Pac J Cancer Prev, 15, 2341-4.

Siraj AK, Al-Rasheed M, Ibrahim M, et al (2008). RAD52 polymorphisms contribute to the development of papillary thyroid cancer susceptibility in Middle Eastern population. $J$ Endocrinol Invest, 31, 893-9.

Suzuki K, Matsubara H (2011). Recent advances in p53 research and cancer treatment. J Biomed Biotechnol, 2011, 978312.

Verit FF, Yucel O (2013). Endometriosis, leiomyoma and adenomyosis: the risk of gynecologic malignancy. Asian Pac J Cancer Prev, 14, 5589-97.

Vijayaraman KP, Veluchamy M, Murugesan P, et al (2012). p53 exon 4 (codon 72) polymorphism and exon 7 (codon 249) mutation in breast cancer patients in southern region (Madurai) of Tamil Nadu. Asian Pac J Cancer Prev, 13, 511-6.

Vineis P (2004). Individual susceptibility to carcinogens. Oncogene, 23, 6477-83.

Wang F, Wang P, Wang B, et al (2014). Association between TP53 Arg72Pro polymorphism and thyroid carcinoma risk. Tumour Biol, 35, 2723-8.

Wu R, Li B (1999). A multiplicative-epistatic model for analyzing inter specific differences in out crossing species. Biometrics, 55, 355-65.

Xia LY, Zeng XT, Li C, et al (2013). Association between p53 Arg72Pro polymorphism and the risk of human papillomavirus-related head and neck squamous cell carcinoma: a meta-analysis. Asian Pac J Cancer Prev, 14, 6127-30.

Xiang B, Mi YY, Li TF, et al (2012). Updated meta-analysis of the TP53 Arg72Pro polymorphism and gastric cancer risk. Asian Pac J Cancer Prev, 13, 1787-91.

Zhou X, Gu Y, Zhang SL (2012). Association between p53 codon 72 polymorphism and cervical cancer risk among Asians: a HuGE review and meta-analysis. Asian Pac J Cancer Prev, 13, 4909-14. 